\title{
Observation of Magnetic and Electric Field in STEM by Using CCD Camera
}

\author{
Akira Yasuhara*, Wataru Inami**, Kazuya Yamazaki*, \\ Akihiro Saitow***, Kimiharu Okamoto*, Hirotoshi Endo*, Motoharu Kawazu*, \\ Toshiaki Suzuki****, Ichiro Ohnishi* and Yukihito Kondo*. \\ * Electron Optics Division, JEOL Ltd., 3-1-2 Musashino, Akishima,Tokyo 196-8558, JAPAN \\ ** Division of Global Research Leaders, Shizuoka University, 3-5-1 Johoku Naka-ku, Hamamatsu, \\ Shizuoka, 432-8561, JAPAN \\ *** Technical Department, JEOL Engineering Co. Ltd., 3-1-2 Musashino, Akishima,Tokyo 196- \\ 8558, JAPAN \\ **** Metrology Inspection Division, JEOL Ltd. , 3-1-2 Musashino, Akishima,Tokyo 196-8558, \\ JAPAN
}

In order to observe magnetic field and electric field with STEM (Scanning Transmission Electron Microscopy), the DPC (Differential Phase Contrast) imaging method [1] has commonly been used. However, the DPC imaging requires a special quadrant STEM detector.

A STEM Diffraction Imaging technique developed by Gatan Inc. has reached a very high level progress in storage capacity and processing speed. This technique has made it possible to acquire a series of electron diffraction patterns on a Slow Scan Charge-Couple Device (CCD) camera for each pixel of the scanning image.

We have developed a new method to observe magnetic field and electric field with conventional Transmission Electron Microscopy (TEM), by using the STEM Diffraction Imaging technique instead of the quadrant STEM detector. This paper reports the method with an example of observed magnetic field. A STEM Diffraction Imaging data set was acquired with a JEM-2100F(TEM/STEM). We extracted magnetic and/or electric information of the specimen with our own program by using the MATLAB from the acquired huge data, which has four dimensions, that is, two dimensions for $\mathrm{x}$ and $y$ coordinates of a map and two dimensions for $u$ and $b$ for each diffraction pattern.

STEM BF image of a Nd-Fe-B film with a conventional scanning detector is shown in (1) in a schematic flow diagram of our method. (2) shows a STEM Diffraction Imaging data of the rectangular area in (1). As shown in (3), an individual electron diffraction pattern for each pixel is divided into four Regions Of Interest (ROI) (A, B, C and D). Images (A, B, C and D) in (4) are reconstructed from the integrated intensity for four corresponding ROIs, respectively. (5) and (6) show the magnetization distribution (vertical and horizontal). For the vertical magnetization distribution shown in (5), the image intensity is calculated by $(A+C)-(B+D)$, which represents the derivate of phase or magnetic potential in the vertical direction. And for the horizontal one the intensity is calculated by $(\mathrm{A}+\mathrm{B})-(\mathrm{C}+\mathrm{D})$ resulting in the horizontal magnetization distribution. Note that an electron beam deflects to a perpendicular direction of the magnetic field. As shown in (5) and (6), we could visualize the magnetic field inside and outside of the specimen by this method. We summarize the advantages for this method as follows;

1. The DPC image can be acquired with the conventional STEM instrument equipped with a CCD camera without using the special quadrant detector

2. Magnetization or di-polarization in any direction can be visualized, by choosing the adequate shapes or dividing into many sector-formed ROIs.

3. Once a full data set is acquired, any type of post-processing can be applicable after the observations. 
References

[1] J. N. Chapman et al., Ultramicrscopy 3, (1978). 203

[2] A. Yasuhara et al., JSM2008 program and proceedings, (2008), 181

[3] A. Yasuhara et al., AMPC9 proceedings, (2008),699

\section{STEM BF image \\ 2. Diffraction Imaging Data Set}

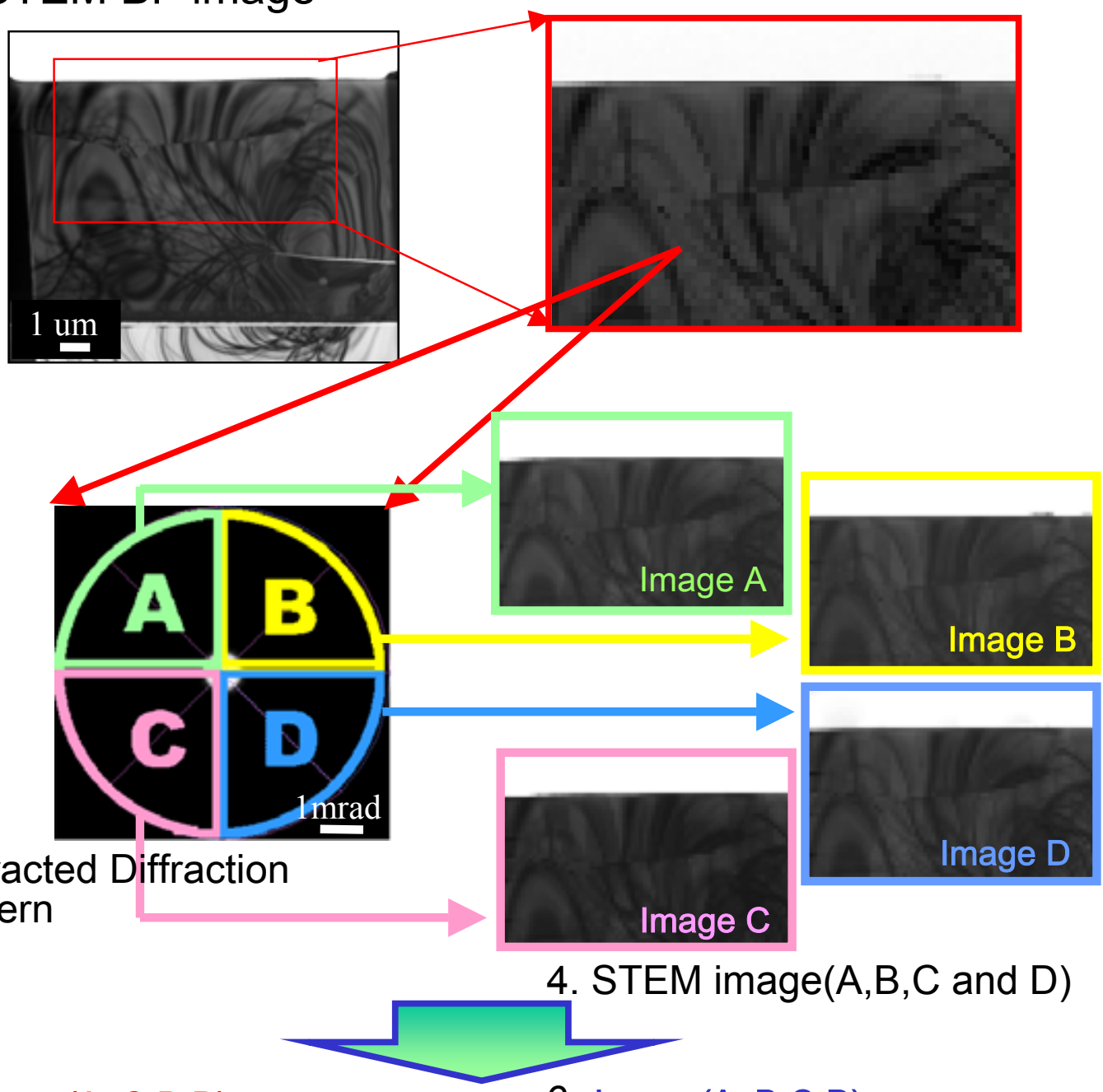

5. Image (A+C-B-D)

6. Image (A+B-C-D)
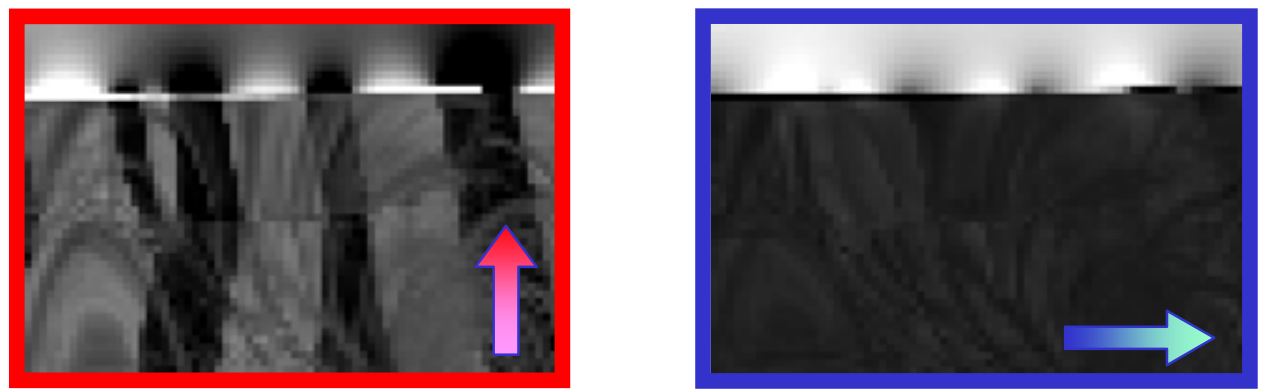

Magnetization distribution (vertical) Magnetization distribution (horizontal)

Figure. The flow chart of the observation of magnetic (electric) field with the DPC method by using the CCD camera in a STEM mode. 